\title{
Infant anaphylaxis: the importance of early recognition
}

\author{
This article was published in the following Dove Press journal: \\ Journal of Asthma and Allergy \\ 3 July 2013 \\ Number of times this article has been viewed
}

\begin{abstract}
A Dosanjh
Department of Pediatrics, Rady Children's Hospital, San Diego, CA, USA
\end{abstract}

Correspondence: A Dosanjh Pediatric Respiratory, 530 Lomas Santa Fe-M, Solana Beach, CA 92075, USA Email pulmd1@gmail.com

\begin{abstract}
Anaphylaxis is an acute severe reaction involving multiple systems that results from a rapid release of inflammatory mediators. Patients with asthma and prior allergic reactions are at risk for anaphylaxis. Infants can present a special challenge, as the hallmark symptoms and signs of anaphylaxis may be mistaken as normal findings. These include drooling, vomiting or diarrhea, scratching, and drowsiness. The clinical manifestations of anaphylaxis are broad, as a result of it being a systemic response to an external agent. Among infants and children, there are often respiratory and cutaneous findings. There also can be subtle signs and symptoms, which can often be missed or the findings misinterpreted as normal for developmental age. The incidence of anaphylaxis has increased globally among children presenting with allergic reactions. Early recognition of the signs and symptoms is crucial to effective diagnosis and treatment. This is particularly true among infants 13 months of age or younger who are nonverbal and may have subtle signs and symptoms of a life-threatening reaction to allergens. The purpose of this article is to highlight the differential clinical presentations of young children with anaphylaxis.
\end{abstract}

Keywords: anaphylaxis, infant, food allergy

\section{Introduction}

Anaphylaxis is a severe and often fatal systemic allergic reaction that occurs after contact with a triggering agent. The risk factors for anaphylaxis include a history of asthma, prior history of reactions, younger or older age, use of angiotensin-convertingenzyme inhibitors, pregnancy, cardiovascular disease, and history of mastocytosis.

Biphasic reactions can occur hours or days after the initial reaction. A history of low baseline peak expiratory flow rates and a prior history of asthma have been reported as risk factors for biphasic reactions.

Anaphylaxis is immunoglobulin E (IgE)-mediated, whereas anaphylactoid reactions are non-IgE-mediated, but both present clinically in a similar manner. The true incidence of anaphylaxis varies, depending on the population, but has been estimated to be between 3.2 and 58.9 cases per 100,000 population. There is evidence of a global increase in anaphylaxis incidence, and this observation parallels the increase in worldwide asthma incidence. ${ }^{1-3}$

\section{Clinical presentation}

The presentation and diagnosis of anaphylaxis primarily relies on the timing of acute and sudden potentially fatal symptoms in relation to exposure to the offending agent. There is typically an acute onset of skin and/or mucosal symptoms, as described in the diagnostic criteria established by the National Institutes of Health. 
Respiratory distress, such as wheezing, stridor, shortness of breath, and/or hypotension, or associated symptoms of end-organ dysfunction (eg, hypotonia, syncope, renal failure, seizure activity, incontinence) occur during the progression of the illness. ${ }^{4}$

\section{Epidemiology}

In an Australian study of fatalities caused by anaphylaxis between 1997 and 2006, there was essentially no change in the number of fatalities reported. The number of admissions, however, increased by $350 \%$ during the same time. Drug-induced and insect sting deaths were more common among the older population. In the analysis of food-related anaphylactic deaths, there were seven food-related deaths, of which all except one were among children 11 years of age or younger. Five of the patients had prior food reactions, and all of the patients had asthma. Peanut allergic reactions were associated with three of the five patients with a history of peanut reactions. ${ }^{5,6}$

In the US registry of anaphylaxis fatalities, there were 32 deaths between 1994 and 1999 resulting from food reactions; young age, delayed use of adrenaline, lack of availability of adrenaline, prior severe reactions, and asthma were identified as risk factors. A high incidence of peanut or tree nut allergy was also observed. ${ }^{7}$

The particular type of food reactions vary with ethnic and regional dietary preferences. In one study of 1110 Italian adults and adolescents with food allergies, $5 \%$ had a history of anaphylaxis. Of these patients, 39 had identifiable causes of anaphylaxis, including peach $(n=13)$, tree nuts $(n=9)$, and shrimp $(n=10) .{ }^{8}$ The most common causes of anaphylaxis among children in the United States are fish, shellfish, milk, soy, wheat, eggs, meat, tree nuts, and peanuts. The type of food reaction is often age-related. Children younger than 7 years were more likely to have a reaction to milk $(n=89)$ compared with older children $(\mathrm{n}=124)$, who were more likely to have higher rates of seafood and peanut reactions, according to results from a study based in New York. In the same study of 192 children, 213 anaphylactic reactions were identified. As a group, seafood accounted for $28 \%$ and peanuts for $20 \%$ of these allergic reactions. ${ }^{9}$

\section{Anaphylaxis and infants: subtle signs and symptoms}

Infants are at higher risk for anaphylaxis and present certain clinical challenges when determining whether there is an anaphylactic reaction. Among infants, the recognition of anaphylaxis may be more difficult because of its wider range of presentations. In a study of children who presented to the Boston Children's Hospital or the Massachusetts General Hospital pediatric emergency departments with food-related allergic reactions between 2001 and 2006, age-related patterns were studied. The study reported that milk allergy was more common among infants and shellfish allergy was more often identified among adolescents. Cardiovascular symptoms were rarely reported as a presenting clinical feature of anaphylaxis among the younger children. ${ }^{10}$ The measurement of blood pressure readings and diagnosis of hypotension though among infants may be underused and underreported. ${ }^{9}$

In the New York study of 213 anaphylactic reactions among 192 children presenting to the Mount Sinai pediatric emergency department between 2004 and 2008, the analysis of infants identified certain challenges in establishing the diagnosis of anaphylaxis. The authors identified the fact that infants are less likely to have a blood pressure measurement obtained in the emergency department compared with older children. Because hypotension is one sign of anaphylaxis, this could lead to underdiagnosis among infants. In the New York study, the authors found that only $16.6 \%$ of the infants studied had a blood pressure measurement, and only $12.5 \%$ of children younger than 3 years had a blood pressure measurement compared with $90 \%$ of patients aged 3 years and older. Other clinical features of anaphylaxis may be more difficult to identify in this age group. A wide variety of presentations includes dysphagia and drooling. ${ }^{9}$ Because teething results in drooling, this physical sign may go undocumented or may be assumed to be unrelated to the allergic reaction. Similarly, pruritus, a physical sign of histamine release, may present as scratching, which may be undocumented.

In a study of Boston emergency departments and children presenting with food-related allergic reactions, age-related differences were described. Among the infants 12 months of age or younger, there were higher rates of food allergy (83\%), and of the food allergens, milk was the most commonly identified (40\%) in the infant age group. Infants also were more likely to present with hives, wheezing, and vomiting compared with older children. ${ }^{10}$

In a case report of a 4-month-old infant with immunologically confirmed cow's milk protein allergy, the authors report that anaphylaxis occurred after ingestion of goat's milk. There was no prior goat's milk exposure. ${ }^{11}$ The report recommended that goat's milk not be used in this setting, as ingestion may elicit an IgE-mediated reaction if there is an immune reaction to cow's milk protein. 
The recommendation of soy formula also has been challenged. A report of anaphylaxis after soy ingestion by a 9-month-old infant with cow's milk allergy describes the problem of formula contamination. ${ }^{12}$ In this report, the infant had severe persistent asthma and positive specific $\operatorname{IgE}$ to cow's milk protein, as well as a negative test to soy protein. The infant was treated with an elimination diet, which eliminated egg, milk, potatoes, and peanuts. A systemic reaction was reported after the infant ingested only $0.4 \mathrm{~mL}$ of cow's milk, which had contaminated the soy formula. Soy formula had been tolerated previously, while the infant was breastfeeding. After the ingestion of a formula contaminated with cow's milk at age 9 months, the repeat specific $\mathrm{IgE}$ levels were higher to both cow's milk and soy $(72.5 \mathrm{kU} / \mathrm{L}$ and $2.3 \mathrm{kU} / \mathrm{L}$, respectively) than initial values obtained on prior testing.

\section{Immunization-related anaphylaxis}

Infants receive numerous vaccinations in the first 2 years of life. Immunizations are not considered to be a significant risk factor for anaphylaxis, but the occurrence has been documented. In one prospective observational cohort study conducted in Ireland from 2006 to 2010, the urban emergency department administered vaccinations on referral for pediatric patients considered at high risk for an allergic reaction. A total of 446 vaccines were administered to 374 patients in the emergency department. Of these patients, 310 received the measles, mumps, and rubella vaccine (MMR) and 64 received a non-MMR vaccine. Suspected egg allergy was the main reason for the majority of referrals for MMR administration (seen in 261 of 310 patients who received the MMR in the emergency department). Among the 446 vaccinations administered, six (1.3\%) resulted in reactions, all of which were minor. The authors concluded that significant concerns related to potential allergic reactions can delay administration of the vaccine and may not be warranted..$^{13}$ In another study conducted in the United Kingdom, Erlewyn-Lajeunesse et al ${ }^{14}$ studied children younger than 16 years in the United Kingdom and Ireland who had suspected anaphylaxis after immunizations. All the cases were reported through the British Pediatric Surveillance Unit between September 2008 and October 2009. More than 5.5 million vaccines were administered during that time. The results indicated that seven children had an anaphylactic reaction, defined as reports submitted by a pediatrician of a severe adverse event following immunization. Six children required intramuscular adrenaline and fluids, but all made a complete recovery. Of the six children treated, all had special circumstances such as receiving a number of vaccines that was higher than usual on the day of the reaction, prior reactions, prior atopic disease, and idiopathic urticaria. Four episodes occurred more than 30 minutes after the immunization. This would suggest that a longer observation period should be instituted. There were no MMR reactions among 750,000 children who received the routine vaccination schedule in the United Kingdom and Ireland. There were two cases of anaphylaxis to a single-component measles vaccine, which was calculated to be an extrapolated incidence of 12.0/100,000. Of the seven cases of anaphylaxis reported, four were level 1, or major reactions, as per the Brighton Criteria. ${ }^{14}$

Reports of latex anaphylaxis among infants raise the possibility that some of the vaccine-related reactions may be related to latex exposure during the administration of the vaccine. In one case series of nine infants aged 12 months or younger, six had a diagnosis of atopic dermatitis, one had asthma, and two had no prior atopic disease. ${ }^{15}$

\section{Anaphylaxis and sudden infant death syndrome}

The association of anaphylaxis and sudden infant death syndrome (SIDS) has been suggested by one study of 40 infants with SIDS, in which their serum analysis was compared with that of 32 non-SIDS infants who died because of a specific cause. The authors reported no differences in specific $\operatorname{IgE}$, total $\operatorname{IgE}$, or constitutive alpha tryptase serum levels. ${ }^{16}$ Beta tryptase levels, however, were significantly higher in the SIDS group, and beta forms of tryptase are secreted during allergic/anaphylactic episodes. These findings raise the possibility that at least some cases of SIDS may be undiagnosed anaphylaxis. A prior study, conducted over 5 years, of 51 patients with SIDS, with a median age of 3 months, showed no significant differences between patients with SIDS and age-matched control non-SIDS infants $(n=13)$ in postmortem levels of allergen-specific IgE or total tryptase levels. The most frequently detected allergen-specific IgE was similar between the two groups. ${ }^{17}$ Both studies revealed that young infants diagnosed with SIDS may have detectable levels of tryptase.

\section{Blood pressure monitoring in the pediatric emergency department}

Hypotension with a temporal relationship to exposure to an offending triggering agent may occur alone or in conjunction with other systemic manifestations of anaphylaxis. It is an important sign of cardiovascular compromise or endorgan dysfunction. According to an online Latin American 
Survey on Anaphylaxis among children and adolescents, there were 191 cases (July 2008 to June 2010) from 14 Latin American countries, including Mexico and Portugal. Children younger than 2 years were more likely to have a food reaction (53.3\% versus $36.1 \%$ ), and most of these were attributable to cow's milk and egg reactions. Cardiovascular symptoms were reported more often in older children, which may in part be a result of the underreporting of palpitations, dizziness, and lack of blood pressure measurements among the youngest children. The survey reported that $34.4 \%$ of the patients had cardiovascular symptoms, but none were listed as being hypotensive. ${ }^{18}$

In a study of Swedish children who were hospitalized for anaphylaxis in 2007, the authors identified hypotension in the records of these children, which was also a sign described by the European Academy of Allergy and Clinical Immunology task force. The authors studied 135 children, 15\% of whom had severe anaphylaxis. The number of these children with hypotension was not specifically reported. ${ }^{19}$ In another study of 73 children with anaphylactic reactions, hypotension was reported in nine cases. In this retrospective study of cases of anaphylaxis in children younger than 18 years from 2001 to 2009 , hypotension was defined as age-specific low systolic blood pressure or as a greater than a $30 \%$ decrease from baseline systolic blood pressure. ${ }^{20}$ These and other studies emphasize the importance of pediatric blood pressure measurement and the recognition of subtle signs and symptoms in establishing the diagnosis of anaphylaxis.

\section{Clinical diagnostic challenges in infant anaphylaxis}

In a Swiss study of 278 food-allergic reactions analyzed according to specific IgE and age, among the infants studied, $10.3 \%$ were diagnosed with anaphylaxis compared with the overall childhood incidence of $4.7 \%$ in this study. Common presentations included a higher rate of skin manifestations and a comparable rate of gastrointestinal symptoms compared with that seen in older children. Infants in this study also were less likely to present with respiratory difficulty $(10.3 \%$ versus $21.4 \%$ in children older than 36 months). None of the children with food allergies in the infant group presented with rhinoconjunctivitis, laryngospasm, or perioral erythema. ${ }^{21}$ In contrast, one case report was that of an infant aged 2 months who developed anaphylaxis shortly after the application of a menthol containing cologne to the face, presenting with acute shortness of breath, cyanosis, and edema of the face, eyelids, and lips. ${ }^{22}$ Common and less-frequent presentations of infant anaphylaxis are shown in Table $1 .{ }^{23}$ 


\section{Conclusion}

The incidence of anaphylaxis in the emergency department setting has increased globally among children presenting with allergic reactions. Early recognition of the signs and symptoms is crucial to effective diagnosis and treatment. This is particularly true among infants 12 months of age or younger who are nonverbal and who may have subtle signs and symptoms of a life-threatening reaction to allergens.

\section{Disclosure}

The author received a medical education grant from Mylan in 2012. The author reports no other conflicts of interest in this work.

\section{References}

1. Koplin JJ, Martin PE, Allen KJ. An update on epidemiology of anaphylaxis in children and adults. Curr Opin Allergy Clin Immunol. 2011;11(5):492-496.

2. Allen KJ, Koplin JJ. The epidemiology of IgE-mediated food allergy and anaphylaxis. Immunol Allergy Clin North Am. 2012;32(1):35-50.

3. Gupta R, Sheikh A, Strachan DP, Anderson HR. Time trends in allergic disorders in the UK. Thorax. 2007;62(1):91-96.

4. Simons FE. Anaphylaxis pathogenesis and treatment. Allergy. 2011; 66 Suppl 95:31-34.

5. Liew WK, Williamson E, Tang ML. Anaphylaxis fatalities and admissions in Australia. J Allergy Clin Immunol. 2009;123(2):434-442.

6. Pumphrey RS, Gowland MH. Further fatal allergic reactions to food in the United Kingdom, 1999-2006. JAllergy Clin Immunol. 2007;119(4): 1018-1019.

7. Bock SA, Muñoz-Furlong A, Sampson HA. Fatalities due to anaphylactic reactions to foods. J Allergy Clin Immunol. 2001;107(1):191-193.

8. Asero R, Antonicelli L, Arena A, et al. Causes of food-induced anaphylaxis in Italian adults: a multi-centre study. Int Arch Allergy Immunol. 2009;150(3):271-277.

9. Huang F, Chawla K, Järvinen KM, Nowak-Węgrzyn A. Anaphylaxis in a New York City pediatric emergency department: triggers, treatments, and outcomes. J Allergy Clin Immunol. 2012;129(1):162-168. e1-e3.
10. Rudders SA, Banerji A, Clark S, Camargo CA Jr. Age-related differences in the clinical presentation of food-induced anaphylaxis. J Pediatr. 2011;158(2):326-328.

11. Pessler F, Nejat M. Anaphylactic reaction to goat's milk in a cow's milk-allergic infant. Pediatr Allergy Immunol. 2004;15(2):183-185.

12. Levin ME, Motala C, Lopata AL. Anaphylaxis in a milk-allergic child after ingestion of soy formula cross-contaminated with cow's milk protein. Pediatrics. 2005;116(5):1223-1225.

13. Cronin J, Scorr A, Russell S, McCoy S, Walsh S, O’Sullivan R. A review of a paediatric emergency department vaccination programme for patients at risk of allergy/anaphylaxis. Acta Paediatr. 2012 Sep; 101(9):941-945.

14. Erlewyn-Lajeunesse M, Bonhoeffer J, Ruggeberg JU, Heath PT. Anaphylaxis as an adverse event following immunisation. J Clin Pathol. 2007;60(7):737-739.

15. Kimata H. Latex allergy in infants younger than 1 year. Clin Exp Allergy. 2004;34(12):1910-1915.

16. Buckley MG, Variend S, Walls AF. Elevated serum concentrations of beta-tryptase, but not alpha-tryptase, in Sudden Infant Death Syndrome (SIDS). An investigation of anaphylactic mechanisms. Clin Exp Allergy. 2001;31(11):1696-1704.

17. Hagan LL, Goetz DW, Revercomb CH, Garriott J. Sudden infant death syndrome: a search for allergen hypersensitivity. Ann Allergy Asthma Immunol. 1998;80(3):227-231.

18. Solé D, Ivancevich JC, Borges MS, et al; for Latin American Anaphylaxis Working Group. Anaphylaxis in Latin American children and adolescents: the Online Latin American Survey on Anaphylaxis (OLASA). Allergol Immunopathol (Madr). 2012;40(6):331-335.

19. Vetander M, Helander D, Lindquist C, et al. Classification of anaphylaxis and utility of the EAACI Taskforce position paper on anaphylaxis in children. Pediatr Allergy Immunol. 2011;22(4):369-373.

20. Silva R, Gomes E, Cunha L, Falcão H. Anaphylaxis in children: a nine years retrospective study (2001-2009). Allergol Immunopathol (Madr). 2012;40(1):31-36.

21. Ferrari GG, Eng PA. IgE-mediated food allergies in Swiss infants and children. Swiss Med Wkly. 2011;141:w13269.

22. Arikan-Ayyildiz Z, Akgül F, Yilmaz S, Ozdemir D, Uzuner N. Anaphylaxis in an infant caused by menthol-containing cologne. Allergol Immunopathol (Madr). 2012;40(3):198.

23. Simons FE. Anaphylaxis in infants: can recognition and management be improved? J Allergy Clin Immunol. 2007;120(3):537-540.
Journal of Asthma and Allergy

\section{Publish your work in this journal}

The Journal of Asthma and Allergy is an international, peer-reviewed open-access journal publishing original research, reports, editorials and commentaries on the following topics: Asthma; Pulmonary physiology; Asthma related clinical health; Clinical immunology and the immunological basis of disease; Pharmacological interventions and

\section{Dovepress}

new therapies. Issues of patient safety and quality of care will also be considered. The manuscript management system is completely online and includes a very quick and fair peer-review system, which is all easy to use. Visit http://www.dovepress.com/testimonials.php to read real quotes from published authors. 